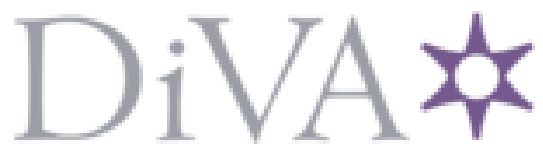

http://www.diva-portal.org

This is the published version of a paper published in .

Citation for the original published paper (version of record):

Åstrand, M., Saarinen, K., Sander Tavallaey, S. (2017)

Surrogate models for design and study of underground mine ventilation

IEEE International Conference on Emerging Technologies and Factory Automation, ETFA, : 1-8

Access to the published version may require subscription.

N.B. When citing this work, cite the original published paper.

Permanent link to this version:

http://urn.kb.se/resolve?urn=urn:nbn:se:kth:diva-298572 


\section{Surrogate Models for Design and Study of Underground Mine Ventilation}

\author{
Max Åstrand \\ ABB Corporate Research \& \\ KTH Royal Institute of Technology \\ Västerås / Stockholm, Sweden \\ max.astrand@se.abb.com
}

\author{
Kari Saarinen \\ ABB Corporate Research \\ Västerås, Sweden \\ kari.saarinen@se.abb.com
}

\author{
Shiva Sander-Tavallaey \\ ABB Corporate Research \\ Västerås, Sweden \\ shiva.sander-tavallaey@se.abb.com
}

\begin{abstract}
Ventilation is vital for the production in an underground mine. Therefore, it is important to have efficient and accurate design tools in order to ensure and optimize the airflows in the mine. There are several commercial software for airflow simulation based on first principles. However, the computational cost of simulation together with integrational obstacles when connecting simulation to control strategies limits the benefit of these tools. In this paper an approach utilizing surrogate models as a complementary design tool is presented. It is shown that using surrogate models one can with rather low computational expense evaluate and benchmark different control strategies. It is also shown that the models can be used for identifying possible bottlenecks in the system in advance. Moreover, the use of surrogate models transfer the simulation into a developmentfriendly environment (such as Matlab). A test case is used based on a real underground mine ventilation design. Two types of surrogate models are fitted to process data; multiple least squares regression and a Gaussian process model. Sensitivity analysis on the surrogate shows the potential of using surrogate models for identifying bottlenecks. Furthermore, the surrogate is used to benchmark two different control strategies for mine ventilation.
\end{abstract}

\section{INTRODUCTION}

\section{A. Problem Description and Motivation}

It is not possible to operate a modern underground mine without a ventilation system which contains many different components. In particular there are often large surface fans which push air through ventilation shafts down to production areas where more fans are used to distribute the air inside the mine. The air is guided by tunnels in which different activities take place (such as excavation). The air is later expelled out on the surface level possibly by means of more surface fans. In many mines dampers are also used to further redirect the air down in the mine.

In mines all around the world it is still common with static (full blow) control strategies with zero level of automation. This means that a fan is set to run at nominal speed and (at best) the air is directed using dampers, causing energy loss. The ventilation strategy is constant no matter what activity is going on (with a possible exception during blasting) and it does not consider where in the mine the activities are actually performed. Removing blast gases or pollutants from e.g. diesel equipment is important to ensure a safe and operable work environment. The energy consumption of ventilation typically comprises around $50 \%$ of the total energy consumption for an underground mine, with fans commonly in megawatts. Techniques for localization of personnel and vehicles in underground mines have existed for many years, and it enables smarter control of the air by focusing the air to where it is needed. A control strategy with focus on an energy-efficient ventilation system will be even more crucial when mines goes deeper, as costs increase exponentially with depth of production [1]. Furthermore, the control system can preferably be used to monitor the ventilation system enabling early detection of anomalies in the production chain, and thereby avoiding unplanned stops caused by lack of air.

Engineers all over the world use simulators for design and optimization of the ventilation system for the continuously evolving mine. Several commercial software exist for this purpose. The rather complex and advanced simulation tools share a common drawback. The models are computationally expensive and not easy to adapt for a fast evaluation of different control strategies and/or identification of bottlenecks in the system during the design phase. A mathematical approach to decrease the computational expense of using physical simulations is to utilize surrogate models. The surrogates can be used as a statistical representation of the more expensive physical simulation.

Surrogate models have been used extensively in engineering design. In the literature the focus is often on some multiobjective design problem which needs to be handled efficiently. For instance in [2] the authors use a surrogate model instead of CFD simulations of hypersonic spiked blunt bodies. In [3] a surrogate model is used for modelling the flow of groundwater, and in [4] they optimize high-temperature superconducting filters. The review [5] describes pro's and con's of different models. The literature on using surrogate models on systems instead of components, as well as surrogate models as a tool of analysis, is more rare. One example of the earlier is [6], while an example of the latter can be found in [7] who study fault detection in robotics.

\section{B. Main Contribution and Outline}

The goal of this paper is to evaluate the possibilities of systematically using surrogate model as a tool for design and 
study of underground mine ventilation systems. An important idea considered here is to show the strength of metamodel approaches such as surrogate models as a complement to sophisticated and advanced design tools. One test case with two different applications have been considered. It is shown that the approach can be used to identify bottlenecks in a system by studying the impact of different process parameters on the process performance. It is also demonstrated that by using surrogates one can rather easily benchmark and choose the most efficient control strategy for the ventilation system.

While Sec. I gives an introduction to the field and the background for the chosen approach, in Sec. II, physical model based design tools as well as surrogate models are briefly explained. Two different control strategies used in this paper are also presented. The building and validation of surrogate models are described in Sec. III. In Sec. IV the simulation models and some results are presented. Finally, the future work and concluding remarks are discussed in Sec. V. The underlying physical simulation engine used in this paper is VentSim [8]. The development is done in Matlab [9] using the SUMO-toolbox [10] and the SAFE-toolbox [11].

\section{Underground Mine VEntilation}

Underground mine operation is a combination of many diverse activities. Excavation is often used to indicate activities related to rock removal such as drilling, charging, blasting and loading. Most activities carried out in an underground mine require different mobile machinery, with the common factor that most of them are still running on diesel. Using combustion engines in a confined environment introduce one of the main objectives for the ventilation system - getting rid of pollutants $(\mathrm{CO} 2, \mathrm{NOx})$ from the mobile machinery. The ventilation system also provides a feasible working environment for both man and machine. In some deep mines, the rock temperature is high $\left(\sim 60^{\circ} C\right)$, in some others the portion of various natural gases like radon and methane is too high, all in all indicating that ventilation is not only needed for continued production but it is also crucial for the bare lives of the personnel. Therefore, a proper design and understanding of ventilation system is an important part for a safe and profitable underground mine operation.

In this section the design tools utilized in this work, i.e. VentSim as well as the workflow of adapting a surrogate model are introduced. Furthermore, two different control strategies, benchmarked in this study, are described.

\section{A. Design Tools}

When simulating mine ventilation one is interested in what airflows are achieved in the mine under certain operational conditions. It is crucial to know how the system will react on anomalies such as partial blockages of airways corresponding to several trucks getting stuck behind each other in the main ramp, or possibly the effect of a faulty damper. Ideally one would like to approach the problem by solving the NavierStokes equation [12], for each and every operational case. However, due to the complexity of an underground mine ventilation system together with the complexity of the NavierStokes equation this is not computationally feasible. There are a number of simulation software tools on the market using different iterative approaches solving the simplified pipe flow problem. In this section Ventsim as the simulation tool used for this work is presented. The developed simulation model is then used as a base for adapting surrogate models.

1) Physical Model/VentSim: The physical simulation of the mine ventilation system is done using the software VentSim which uses the Hardy Cross algorithm [13] for estimating pipe flows. VentSim is the industry standard for simulation of underground mine ventilation [14], and it is used by some 1000 mines and consultants around the world. Furthermore, mining companies often supports VentSim models by real site calibrations which is a prerequisite for accurate simulations. Obstruction of airways are introduced by using the obstruction feature in VentSim. Doors and openings are controlled by modifying the standard values for the total resistance of a closed door to values corresponding to a similar airway without a door. The effect of surface air temperature and moisture content of air is not considered, even though in principle it could be included since VentSim supports this. Another effect which has not been considered in the simulation is the thermodynamic effect of heat transfer from the rock to the air, possibly leading to natural drafts. This information can also be included in VentSim simulations, but the recommendation from the manufacturer is that it should only be included if thermodynamic parameters are well known (e.g. heat transfer coefficient). Since the models used have been calibrated onsite without these effects taken into consideration, this was not included in the simulation either. Note that simulations are performed by a steady state algorithm, hence the solutions obtained do not include the dynamics in the system.

2) Surrogate Model Approach: The general idea of using surrogate models is to develop a "good enough" model for evaluation of the ventilation system in an underground mine. This data-driven modelling approach requires a rather accurate simulation model generating enough and relevant data for modelling purposes. In this work the calibrated VentSim models have been used as simulation engine. The idea is simply to model the process as:

$$
\boldsymbol{y}=\left[\begin{array}{lll}
\Delta P & \boldsymbol{E} & \boldsymbol{Q}
\end{array}\right]^{T}=\hat{f}\left(\left[\begin{array}{lll}
\boldsymbol{\beta} & \boldsymbol{R} & \boldsymbol{O}
\end{array}\right]^{T}\right)=\hat{f}(\boldsymbol{x}) .
$$

Here the output vector $\boldsymbol{y}$ is thus composed of a vector of differential pressures over fans $\Delta P$, a vector of power consumptions of fans $\boldsymbol{E}$ and a vector of airflows in the mine $\boldsymbol{Q}$. The considered flows are any flows in the mine where an airflow measurement device exists. The input vector $\boldsymbol{x}$ contains a vector of control signals to the fans $\boldsymbol{\beta}$, speed, as well as two discrete vector variables $\boldsymbol{R}$ and $\boldsymbol{O}$ modelling anomalies in the mine. The components in $\boldsymbol{R}$ can take two distinct values whether or not doors in the mine are open or closed. Components in $\boldsymbol{O}$ models the obstruction of certain airways. The ventilation design is such that the doors should be closed for the air to take the path that is intended. By changing $\boldsymbol{R}$ 
and $\boldsymbol{O}$ we can thus model the mine both with and without anomalies inserted.

\section{B. Control Strategies}

An intuitive improvement over a static (full blow) ventilation strategy is to use a method known in the industry as Ventilation-On-Demand (VOD). This approach uses localization and linear modelling to control fans according to the need of the localized assets. Another possible approach is a Model Predictive Control (MPC) formulation which not only delivers the required airflow, but it also provides a mean of doing so while minimizing power consumption. Although not the main focus in this work these two control strategies will be briefly introduced, and will further-on be called $V O D$ and $M P C$.

1) VOD: A common way of implementing VOD is based on modelling the airflow as a linear function of the required speed in an open-loop fashion. Assume that the flow $Q_{100}$ delivered by a fan running at $100 \%$ under certain operating conditions is known. Under the linearity assumption, the flow delivered at fan speed $\beta$ relative to the nominal speed is then given by $Q=\beta Q_{100}$. This means that in order to obtain an open-loop control in which the delivered flow ideally is in the vicinity of the required airflow, $Q_{r e q}$, the fan speed can be set to

$$
\beta=\frac{Q_{r e q}}{Q_{100}} .
$$

The main issue with this approach is that airflow is also dependent on differential pressure over the fan. Thus, we do not know what airflows we are actually achieving. It is possible that some of these fans are operating in regions of stalling or low-pressure leading to highly reduced performance. Despite the drawbacks, VOD provides obvious improvements over the static control strategy and is considered best-practice in large parts of the mining community [15].

2) $M P C$ : Ventilation-on-demand neglects the interaction between the flows. To increase the flow in one part of a mine, the flow might decrease in other parts. With respect to the power consumption, the ideal control operation might require a complex combination of fan speeds. This statement is especially true when considering complicated mine topologies where the interaction between flows is significant. The implementation described here is based on [16].

Let $\boldsymbol{Q}=\left(Q_{1}, \ldots, Q_{n}\right)$ be a vector of flows in an underground mine. As long as the ventilation system is non-trivial then for a given demand of flows $\boldsymbol{Q}_{r e q}=\left(Q_{r e q, 1}, \ldots, Q_{r e q, n}\right)$ it exists more than one way of directing the air such that $\boldsymbol{Q} \succeq \boldsymbol{Q}_{\text {req. }}$. Several methods to achieve the same goal implicitly implies a possibility for optimization.

Ideally one would like to have a function $\boldsymbol{Q}=f(\boldsymbol{\beta})$ describing the dependence between the airflows in the mine and the vector of control signals $\boldsymbol{\beta}=\left(\beta_{1}, \ldots, \beta_{p}\right)$. Consider a linearization around $Q_{0}$ with deviation $\Delta \beta$

$$
\boldsymbol{Q} \approx \boldsymbol{Q}_{0}+H_{Q} \Delta \boldsymbol{\beta}
$$

where $H_{Q}$ is the Jacobian.
A change of flow $\Delta Q=Q-Q_{0}$ can thus be approximated by $\Delta \boldsymbol{Q} \approx H_{Q} \Delta \boldsymbol{\beta}$. If the solution to $\Delta \boldsymbol{Q} H_{Q}^{-1}$ is not unique, it is beneficial to formulate this as an optimization problem to minimize the power consumption

$$
\begin{array}{ll}
\underset{\beta}{\operatorname{minimize}} & E_{\text {tot }}(\boldsymbol{\beta}) \\
\text { s.t. } & \Delta \boldsymbol{Q} \preceq H_{Q} \Delta \boldsymbol{\beta} \\
& \boldsymbol{\beta}_{\min } \preceq \boldsymbol{\beta} \preceq \boldsymbol{\beta}_{\max } \\
& \boldsymbol{\Delta} P_{\min } \preceq \boldsymbol{\Delta} P \preceq \boldsymbol{\Delta} P_{\max }
\end{array}
$$

where $E_{t o t}$ is the sum of all fan power consumptions. The first set of constraints ensure that the change of control signals have the desired effect, i.e. the required change of airflow is achieved. The second set of constraints limit the control signals to allowed values, while the last set of constraint ensure that the fans are not in regions of stalling or low pressure. Thus, by linearizing and estimating $E_{t o t}$ similar to eq. (3) an incremental MPC controller can be implemented based on eq. (4).

\section{Building And VAlidating SurRogate Models}

\section{A. Test Case}

A test case based on a real underground mine ventilation design is used. A simplified schematic representation of the ventilation design can be seen in fig. 1 . The design consists of three levels, where excavation takes place at each level. The monitored airflows are the flows in each level $\left(Q_{1, \ldots, 3}\right)$, as well as the two "leak-flows" between the levels and the airflow going out from level $3\left(Q_{4, \ldots, 6}\right)$. The fans considered are the surface fan $\left(\beta_{1}\right)$ together with 6 smaller fans $\left(\beta_{2, \ldots, 7}\right)$. Additionally, two doors $\left(R_{1,2}\right)$ and two obstructions $\left(O_{1,2}\right)$ are controllable. Note that fig. 1 only represents a high-level schematic, not the entire complexity of the ventilation design. In total the installed power is in the vicinity of $3 \mathrm{MW}$.

Concerning the dimensions of the surrogate model in eq. (1) the output $\boldsymbol{y}$ is thus a vector with 20 components ( 7 differential pressures, 7 power consumptions, and 6 airflows). The input $\boldsymbol{x}$ contains 7 control signals and 4 anomalies corresponding to a vector with $7+2+2$ components.

\section{B. Surrogate Modelling}

The general idea is to model $\boldsymbol{y}=\hat{f}(\boldsymbol{x})$ as in eq. (1). A common approach is to fit a different surrogate model on each component in the response vector, hence the problem decomposes into approximating numerous scalar outputs $y_{i}$ (denoting one component in $\boldsymbol{y}$ ) as a function of the input vector $\boldsymbol{x}$. In this paper we present results for two surrogate models 1) multiple linear regression model and 2) Gaussian process model. The reason for choosing a linear regression is due to the wide acceptance and understanding of the model in the research community. The Gaussian process is an appropriate choice for the cases where the underlying function is non-linear with a moderate number of input variables [5]. Futhermore, Gaussian processes are mathematically equivalent to many other well known models, including Bayesian linear models, spline models, large neural networks (under suitable 


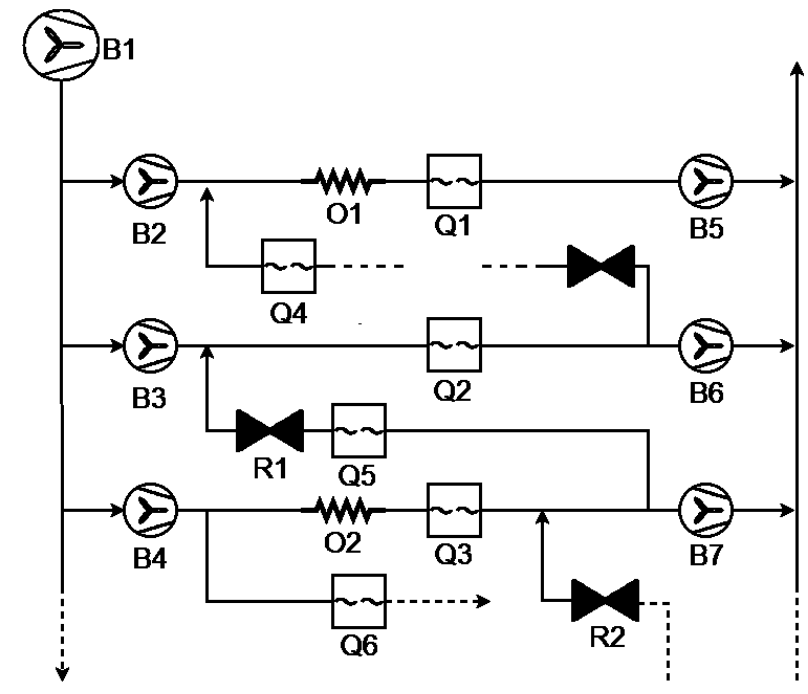

Fig. 1: The ventilation design in the test case where excavation occurs in three horizontal levels which requires ventilation. The considered quantities include fans $(B)$ and airflows $(Q)$, as well as introducable anomalies representing open doors and obstructed airways (labelled $R$ and $O$ respectively).

conditions), and are closely related to others, such as support vector machines [17]. Also, the models may be easier to handle and interpret than their conventional counterparts, such as e.g. neural networks.

1) Multiple Linear Regression: A straight forward and easy to understand approach for constructing a surrogate is by using multiple linear regression model where the outputs are linear combinations of the inputs. In our first simple model we use a polynomial regression function with degree up to cubic terms and with first order interaction terms

$$
\begin{aligned}
y & =b_{0}+\sum_{i=1}^{p} b_{i} x_{i}+\sum_{i=1}^{p} b_{i i} x_{i}^{2}+\sum_{i=1}^{p} b_{i i i} x_{i}^{3} \\
& +\sum_{i=1}^{p} \sum_{j=i+1}^{p} b_{i j} x_{i} x_{j}
\end{aligned}
$$

which can be written in matrix notation as $\boldsymbol{y}=\boldsymbol{X} \boldsymbol{b}$ where $\boldsymbol{X}$ is the data matrix including the linear, squared, cubic, and interaction terms. The regression coefficients $\boldsymbol{b}$ are estimated by solving the normal equation

$$
\hat{b}=\left(X^{T} X\right)^{-1} X^{T} y
$$

2) Gaussian Process Model: A Gaussian process (GP) is completely defined by its mean function and covariance function. Defining the mean function $m(\boldsymbol{x})$ and the covariance function $k\left(\boldsymbol{x}, \boldsymbol{x}^{\prime}\right)$ of the real process $f(\boldsymbol{x})$ as

$$
\begin{aligned}
m(\boldsymbol{x}) & =E(f(\boldsymbol{x})) \\
k\left(\boldsymbol{x}, \boldsymbol{x}^{\prime}\right) & =E\left((f(\boldsymbol{x})-m(\boldsymbol{x}))\left(f\left(\boldsymbol{x}^{\prime}\right)-m\left(\boldsymbol{x}^{\prime}\right)\right)\right)
\end{aligned}
$$

the Gaussian process can be formulated as $f(\boldsymbol{x}) \sim G P\left(m(\boldsymbol{x}), k\left(\boldsymbol{x}, \boldsymbol{x}^{\prime}\right)\right.$.
There exists a large number of applicable covariance functions with free hyperparameters. By far the most common choice is the Gaussian covariance [5]

$$
k\left(\boldsymbol{x}, \boldsymbol{x}^{\prime}\right)=\sigma^{2} \exp \left(-\frac{1}{2}\left(\boldsymbol{x}-\boldsymbol{x}^{\prime}\right)^{\prime} P^{-1}\left(\boldsymbol{x}-\boldsymbol{x}^{\prime}\right)\right)
$$

where $\mathrm{P}$ is a diagonal matrix with diagonal elements $\ell_{1}^{2}, \ell_{2}^{2}, \ldots, \ell_{p}^{2}$. The hyperparameters in the model are signal variance $\left(\sigma^{2}\right)$ and correlation lengths (the $\ell$ 's). It can be shown that the squared exponential covariance function corresponds to a Bayesian linear regression model with an infinite number of basis functions [17].

The hyperparameters can be determined by fitting a GP model to observed sample points by maximizing the marginalized likelihood. Since in our case the response values y are created by computer simulations, there is no measurement error. Therefore, the response values corresponding to new inputs should be predicted excluding the terms corresponding the measurement errors, see [17].

3) Design of Experiment: There is a subtle conceptual difference between the design of a physical experiment and the design of a experiment conducted in a computer simulation. Remember that we are modelling $y=f(\boldsymbol{x})+\varepsilon(\boldsymbol{x})$, where $\varepsilon(\boldsymbol{x})$ contains everything that is not included in $f(\boldsymbol{x})$. Classical Design of Experiment (DOE) techniques [18], such as Central Composite Designs (CCD), are developed for physical experiments to minimize the number of tests and the effect of the random error $\varepsilon(\boldsymbol{x})$ on the estimated parameters. When conducting experiment in a deterministic simulation there is no random error, i.e. conducting the same experiment twice yields the same results. The error term $\varepsilon$ is thus only due to lack-offit, and does not in general have a specific distribution. This means that classical test statistics testing the significance of $\varepsilon$ may be inapplicable [19]. Additionally, the classical designs are typically spread out towards the extremes in design space - leaving holes in the experiment design in the region of most interest. Consider a common CCD design. Projected down onto each coordinate the experiments only evaluate a variable for three values, what goes on in-between is unknown. A sampling technique called Latin Hypercube Sampling (LHS) is a space-filling design which distributes the sample points along each coordinate uniformly. A common procedure is to generate a number of LHS-designs, and use the design which has the lowest correlation among the sample points.

When building a surrogate model for the test case our input to VentSim consists of continuous control signals and the discrete anomaly variables. The discrete variables have 2 levels each; doors can be open or closed, and either there is an obstruction or it is not. A LHS design is constructed for each combination of the discrete variables (4 variables with 2 levels each implies $2^{4}=16$ combinations). VentSim was used to obtain the output data needed for training the surrogate models.

4) Model Validation: A measure is needed to estimate how well the surrogate approximates the true function. A common 

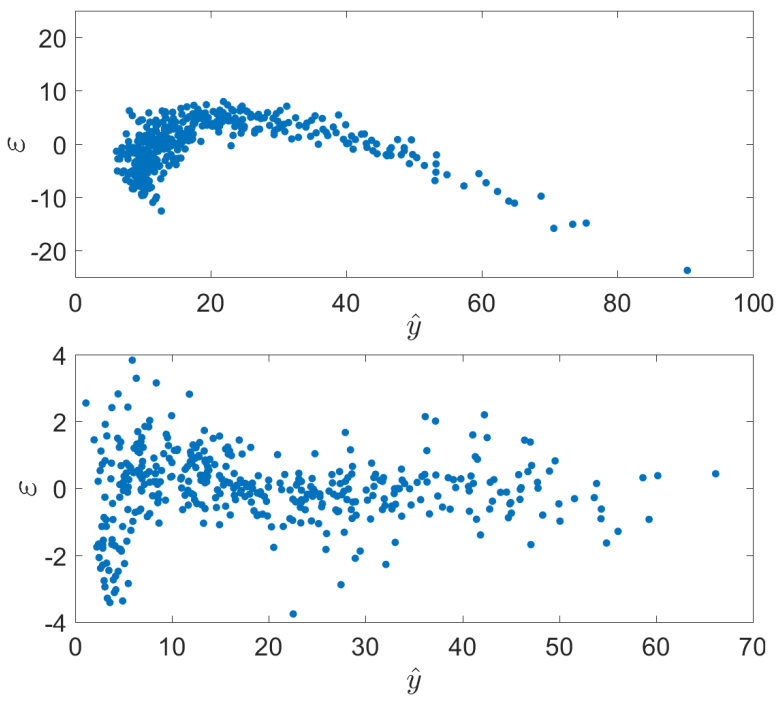

Fig. 2: The prediction residuals for airflow $Q_{4}$ on a test set. The top plot corresponds to the regression model, the second row to the GP model. Note the different scales on the axes.

error measure is the Root Relative Square Error $(R R S E)$

$$
R R S E=\sqrt{\frac{\sum_{i=1}^{n}\left(y_{i}-\hat{y}_{i}\right)^{2}}{\sum_{i=1}^{n}\left(y_{i}-\bar{y}\right)^{2}}},
$$

which is closely related to the well known coefficient of determination (R-squared) from univariate statistics. The $R R S E$ thus measures how much better the model is compared to the simplest of models i.e. the mean.

The concept of overfit, traditionally found in literature when fitting a model to noisy data, does not apply in the same sense in this context. From the surrogates point of view the experiment is the reality, thus all sample points should be interpolated and it is in general not correct [5] to have a smoothing model such as a least squares regression. The absence of noise means that the common concern of modelling the noise instead of the function does not have an intuitive interpretation. It is however important to validate that the surrogate model behaves well between the sample points since its intended purpose is prediction. This is studied by 5 -fold crossvalidation, and RRSE is calculated for each fold [20].

After the VentSim simulations the surrogates were trained and crossvalidated RRSEs was calculated using the SUMOtoolbox [10]. Results for the two surrogate models are

$$
\begin{aligned}
R R S E_{C V}^{(r e g)} & =0.13 \\
R R S E_{C V}^{(g p)} & =0.060 .
\end{aligned}
$$

Additionally, the prediction residuals of the models were examined on a test set. A selection is depicted in fig. 2 where the prediction errors for $Q_{4}$ can be studied. It can be noted that there is some dependence that the regression model does not capture since there is a clear trend left in the residuals.
The low value of $R R S E_{C V}^{(g p)}$ together with inspection of residual plots indicate that the Gaussian process model adequately approximates the underlying simulation.

\section{Simulation And Result}

As examples of what the surrogate can be used for two possible applications are demonstrated in a test case based on a real underground mine. A large part of the ventilation performance originates from the original ventilation system design. Therefore, one demonstration of using surrogate models is when studying the ventilation design of a mine ventilation system by sensitivity analysis of the surrogate. Second, the surrogate is exploited to develop, test, and determine the performance of using different control strategies for the underground mine ventilation.

\section{A. Sensitivity Analysis}

Consider an unknown function with a set of inputs and a single output. It is often interesting to know which of the inputs have a significant effect on the output. Sensitivity analysis [21] denotes methods for examining the effect a certain input has on the output. The goal for ventilation could be to answer questions like what parameters have effect on one particular airflow?

Three methods for sensitivity analysis are in this section introduced; a study of regression coefficients, a study of the kernel parameters of a Gaussian process model, and a perturbation approach known as Morris One-at-a-Time. As with all statistical methods, if it exists multiple choices of achieving the same goal it is often best to evaluate several choices and draw conclusions based on all methods.

1) Regression-Based Analysis : A simple way of sensitivity analysis is to use a least-squares regression, but fitting the regression on normalized inputs so that inputs of different magnitudes can be adequately compared. If the regression coefficients in eq. (6) are rescaled with regard to the largest coefficient

$$
\tilde{b}_{k}=\frac{\hat{b}_{k}}{\max _{j}\left|\hat{b}_{j}\right|},
$$

then the $\tilde{b}_{k}$ 's can be regarded as a sensitivity measure. The $\tilde{b}_{k}$ 's are thus related to the effect that the $k$ 'th variable has on the output.

Care must however be taken when interpreting (13) due to the fact that input variables might be included in several regression terms. For instance, for a single input variable it might exist coefficients for terms of various power (as well as possibly several interaction terms) all relating the impact of an input variable on the output. However, if all the coefficients pertaining to a specific input are small then that input will probably have a small impact on the output, so by examining the variability of the normalized coefficients unimportant variables can be screened. A simple selection rule to determine if the coefficients show enough variability is to select the inputs which have coefficient-variance larger than one standard deviation of all coefficients. 
2) Kernel Analysis of Gaussian Processes: In view of the correlation structure in eq. (9) one might ask what the correlation lengths $\left(\ell_{k}\right)$ really represent. In the univariate case the covariance reduces to

$$
k\left(x, x^{\prime}\right)=\sigma^{2} \exp \left(-\frac{\left(x-x^{\prime}\right)^{2}}{2 \ell}\right) .
$$

It is then clear that $\ell$ is simply a characteristic length of interaction between the samples. Generalizing to the multivariate case $\ell_{k}$ can be interpreted as the characteristic length of interaction along the $k$ 'th dimension. That is, if $\ell_{k}$ is small then the samples along the $k$ 'th dimension have small covariance. Introducing $\tilde{\theta}_{k}$ as the normalized inverse of $\ell_{k}$

$$
\tilde{\theta}_{k}=\frac{\ell_{k}^{-1}}{\sum_{j} \ell_{j}^{-1}}
$$

then small $\tilde{\theta}_{k}$ implies almost constant output, i.e. the input $k$ does not have a large impact on the output. After fitting the GP model we can thus construct and sort the $\tilde{\theta}$ and find out which variables seem to have most effect on the output. It is important also here that the input variables are normalized before fitting to ensure that the variables can be compared even though they differ in magnitude. A similar method can be found in [17] coined Automatic Relevance Detection.

3) Morris One-at-a-time: Another common method for sensitivity analysis is denoted Morris One-at-a-time (MOAT) [22]. The idea is to perturb one input while keeping the other inputs constant and calculating the effect of the perturbation (denoted the Elementary Effect). This is repeated for a number of times on all input values and the combined effects of all perturbations are studied. The elementary effect $E E$ of variable $k$ is calculated as

$$
E E_{k}\left(\boldsymbol{x}_{0}\right)=\frac{1}{\delta}\left(f\left(\tilde{\boldsymbol{x}}_{\mathbf{0}}\right)-f\left(\boldsymbol{x}_{0}\right)\right)
$$

where $\boldsymbol{x}_{0}$ is a point in input space and $\tilde{\boldsymbol{x}}_{\boldsymbol{0}}$ has a small perturbation $\delta$ in one component. After calculating the $\mathrm{EE}$ for many initial points in design space two statistics are studied - the mean value and the standard deviation. The mean value of $E E_{k}$ is the average effect that variable $k$ has on the output. In order to deal with cancellations when calculating the average (due to possible non-monotonicity of the function) the absolute value is often used. The standard deviation of the EE's corresponding to a certain input can be regarded as a measure of non-linearity [23].

4) Evaluation: Sensitivity analyses are conducted using the surrogate models representing the test case showing the strength of using surrogate models as a design tool. The considered inputs are control signals to fans, doors, and possible obstructions, and the outputs are chosen to be the airflows in level 2 and 3 (see fig. 1). The analysis of the regression coefficients in fig. $3 \mathrm{a}$ indicates that only $\beta_{6}$ and $\beta_{7}$ have a significant impact on the airflow in level 2. However, the airflow in level 3 seems to be dependent on more variables including not only the linear coefficients but also interaction terms. Likewise, in fig. $3 b$ the parameters of the kernel in the Gaussian process indicate a similar behaviour where most variability is attributed to control signals $\beta_{5}, \beta_{6}, \beta_{7}$ for level 2 while for level 3 the parameters exhibits a more complex structure. Using MOAT on the Gaussian process surrogate (chosen because it showed best fit) we can elaborate a bit further. The control signal $\beta_{6}$ has the highest elementary effect on the airflow in level 2, but it also has a significant variability. This indicates that the effect of $\beta_{6}$ is both large and non-linear. Concerning the airflow in level 3 the mean and variability of the elementary effects indicate that there are many influential variables including both control signals, doors, and obstructions.

A general observation stemming from all methods concerning the airflow in level 2 is that $\beta_{6}$ is the most influential variable, followed by $\beta_{7}$. Interestingly, by the looks of the schematic reduction of the system in fig. 1 intuition might falsely lead one to believe that $\beta_{3}$ would have a larger impact than $\beta_{7}$. Control strategies based on that intuition fail to recognize that in reality the exhaust fan on the level below have a higher impact than the intake fan at the same level. The non-linearity of the airflow in level 2, as supported by MOAT, also supports the use of a more advanced control strategy since using too simple strategies (such as linear modelling of flow w.r.t. control signal) might be inappropriate. Regarding the airflow in level 3, a general observation is that the airflow has lots of influential variables which also appear to be nonlinear and exhibit interaction effects between different control signals.

This example of applying sensitivity analysis on surrogate models demonstrate one potential use of surrogate modelling in ventilation engineering. The analysis gave insight into which variables actually affect a considered airflow, and cases where found where simple control strategies probably do not operate efficiently.

\section{B. Performance of Control Strategies}

Another possible use of a surrogate model is as a development platform. This enables simulation of days, or weeks, of ventilation performance with minute sampling time which would not be feasible using a physical simulation due to the computational expense.

Since the GP surrogate shows best fit for the test case, this model is used as a base-line for constructing a development platform for the control of the ventilation system. For each measured airflow approximate first order dynamics are introduced. To mimic a real mine setting the time constants of the airflows are set to 10 minutes. The time constants of differential pressures and power consumptions are set to 3 minutes. To simulate the presence of normally occurring fluctuations Gaussian noise is added to the output variables in reasonable magnitudes $\left(10 \mathrm{~Pa}^{2}, 1 \mathrm{~kW}^{2}\right.$, and $\left.5\left(\mathrm{~m}^{3} / \mathrm{s}\right)^{2}\right)$.

The set points for the ventilation (i.e. the required airflows) are based on the presence of activities on different levels in the test case. The number of simultaneous activities in each level is randomly chosen between 0 and 10. Furthermore, each activity is 120 minutes long and demands $14 \mathrm{~m}^{3} / \mathrm{s}$. The 


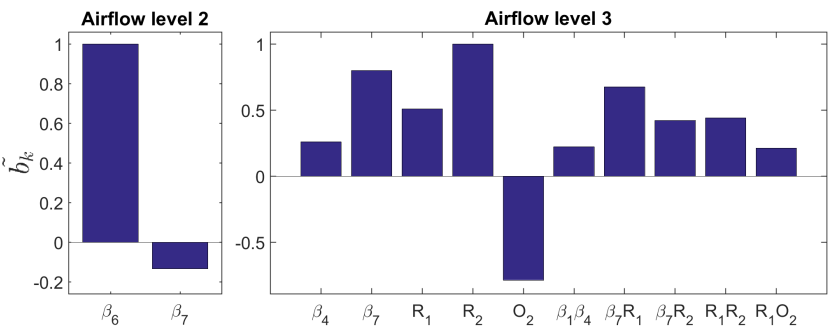

(a) Normalized regression coefficients
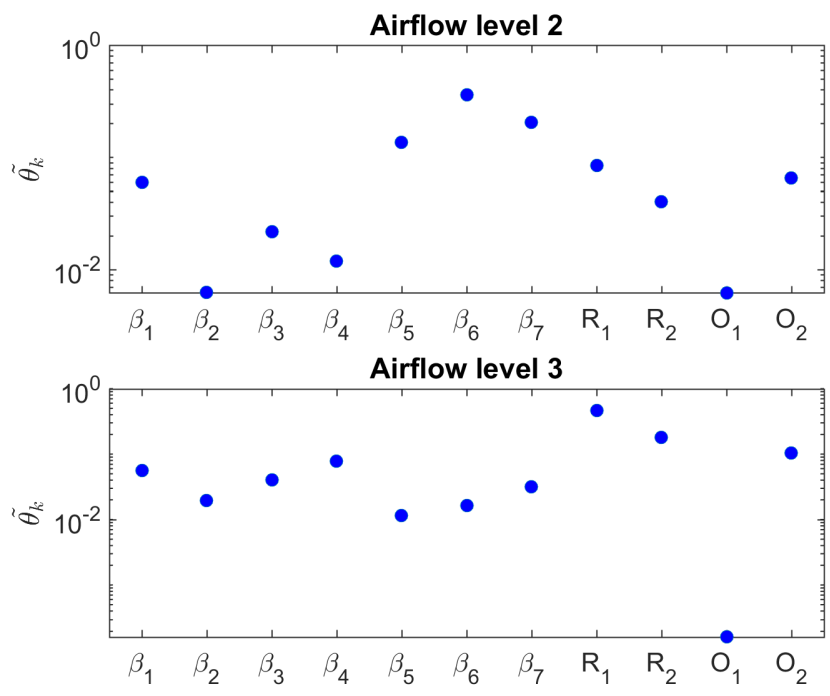

(b) Kernel analysis of Gaussian process model
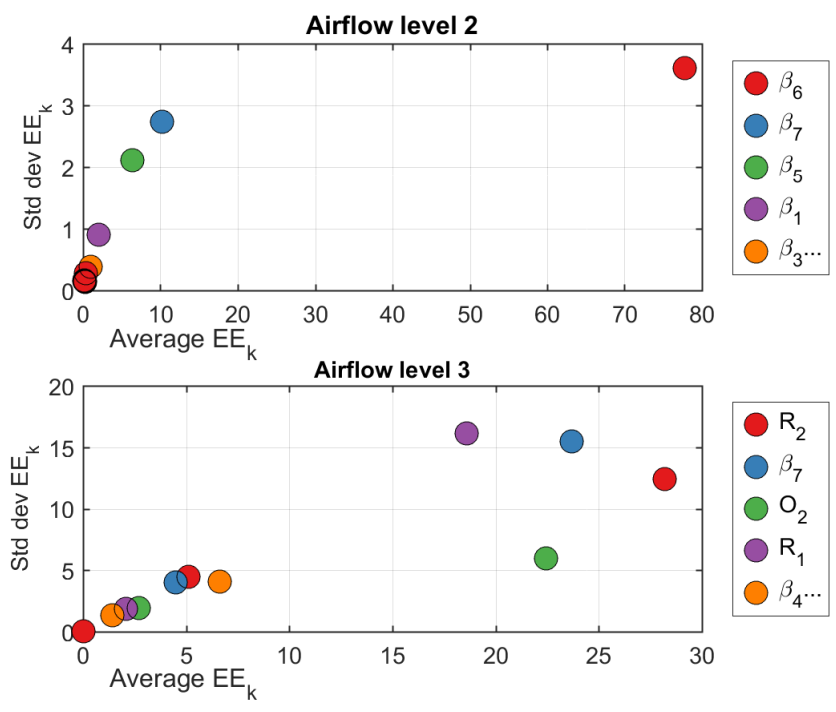

(c) Morris One-at-a-time

Fig. 3: Sensitivity analysis of the airflows on level 2 and 3 in the test case ventilation design.

demand has a lower limit of $45 \mathrm{~m}^{3} / \mathrm{s}$ (a lower limit is often found in reality to prevent the air from being completely still leading to collapsed airducts and reversed airflows). In the VOD implementation if air is needed on a particular level both the intake fan and the exhaust fan on that level are set
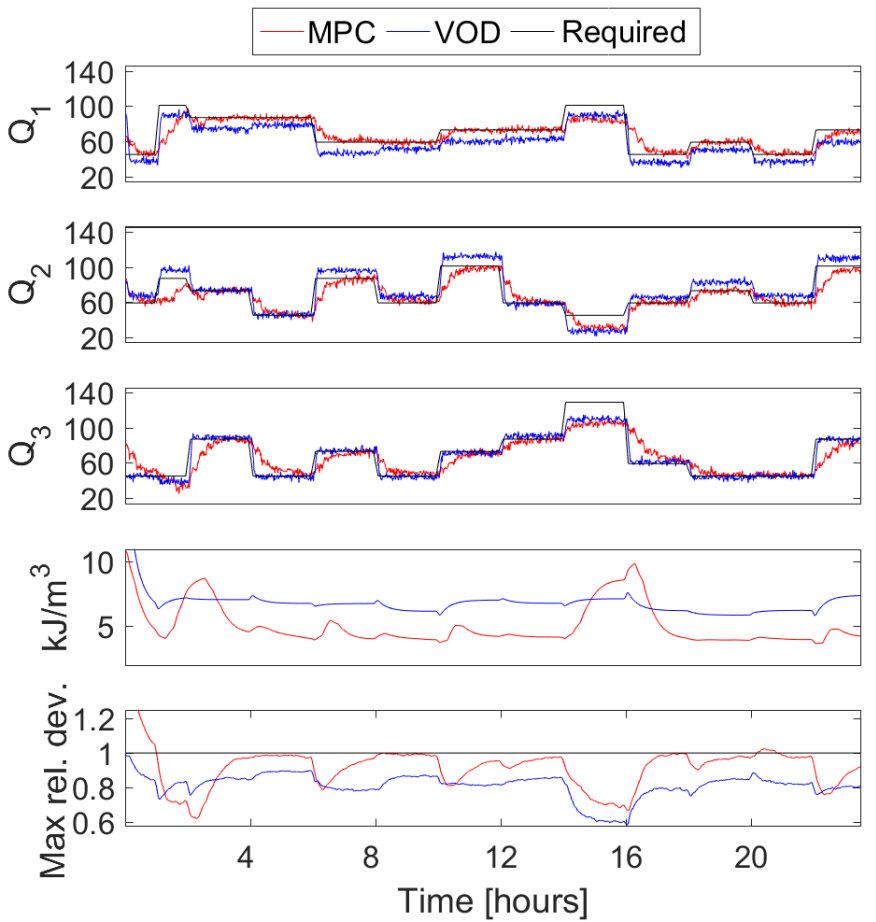

Fig. 4: The result of using different control strategies. The top three plots show the resulting airflows (in $\mathrm{m}^{3} / \mathrm{s}$ ), the fourth plot shows the power consumption per cubic meter air (in $k J / m^{3}$ ), and the bottom plot depicts the maximum relative deviation.

to run at calculated speeds, however the surface fan is not included in the VOD strategy. The MPC model is sampled each 15 minutes.

In the top three plots of fig. 4 we can see the airflows obtained by the two different controllers. It is remarkable that, despite its simplicity, VOD performs quite well as measured by the delivered air. The open-loop property is however notable when considering the excessive airflow in level 2 and the insufficient amount of air in level 1. The MPC controller follows the set points more closely, however it does not adapt as fast to changes in set point.

The MPC controller is more energy efficient. This can be seen in the fourth plot in fig. 4 , where the energy consumption per cubic meter of the MPC controller is compared to VOD. The bottom plot in fig. 4 shows the maximum relative deviation from the required flow. It is evident that the MPC controller is not only more efficient in terms of energy consumption, but it also delivers airflows closer to the demand.

During the hours 14 - 16 neither of the control strategies perform well. High demand at level 1 and level 3 simultaneously thus seems to be hard for the strategies to achieve. A possible explanation could be that it is not physically possible to achieve the required airflows i.e. the system cannot be controlled such as to reach the desired state. 


\section{CONCLUSION}

We have in this paper utilized surrogate models as a mean for analysing the ventilation system in an underground mine. The benefits of using a surrogate is two-fold. It reduces the computational expense, enabling quick prototyping and extensive benchmarking, as well as it moves the potential of the physical simulation from a commercial software into a more development-friendly environment. Through careful experimental design, two surrogates were trained on data collected from a commercial simulation software. It is important to realize that we are operating under two levels of abstractions. The first level is going from reality to physical simulation. The second level is going from physical simulation to statistical model. Other methods for constructing surrogates exist. However, in this case improving the surrogates model fit (which for the Gaussian process model showed mean $R R S E_{C V}$ of $6 \%$ ) is not focusing on the right thing. What is important is the combined error between reality and the surrogate model, and it is likely that the biggest discrepancy comes from the first level of abstraction; going from reality to physical simulations.

The potential use of the surrogate models for optimizing the design and control of mine ventilation systems were demonstrated in two examples. First three sensitivity analyses were introduced to identify possible bottlenecks and design issues in the ventilation system. No large discrepancy was found between the results of using the different methods of sensitivity analysis which facilitates drawing conclusion based on the results. It can be noted that the MOAT method have an advantage over the other methods as it can be used regardless of underlying surrogate. Additionally, it provides a quick way of analysing potential non-linearity. The conducted sensitivity analysis highlighted some dependencies which would be nontrivial to discover using conventional methods.

Second, a surrogate is used to benchmark two control strategies. The implementation of a surrogate model augmented with dynamics and noise used as a development platform was successful. The benchmark showed that the MPC controller more closely follows the required airflows compared to VOD. Additionally, the MPC controller does so with lower energy consumption. However, the benchmark is a demonstration - i.e. in no way conclusive. Additional properties such as stability need to be assessed to ensure a safe deployment.

Both demonstrations show a valuable use of surrogate models for ventilation design and control optimization. Nevertheless, there is a potential to further study the general applicability of the methodology on different mine topologies. For future work it would be interesting to study the application of a similar approach but to another industrial system. It would also be interesting to study the use of surrogate models to glue together different kinds of physical simulations describing different parts of a process.

\section{ACKNOWLEDGMENT}

This work was partially supported by Wallenberg Autonomous Systems and Software Program.

\section{REFERENCES}

[1] J. Parreira, Z. Mullard, J. Meech, and M. G. Vsquez, "How automation and key performance indicators contribute to sustainable development in the mining industry," Second International Conference on Multinational Enterprises and Sustainable Development, 2009.

[2] M. Ahmed and N. Qin, "Comparison of response surface and kriging surrogates in aerodynamic design optimization of hypersonic spiked blunt bodies," 13th Aerospace Sciences and Aviation Technology, 2009.

[3] M. Asher, B. Croke, A. Jakeman, and L. Peeters, "A review of surrogate models and their application to groundwater modeling," Water Resources Research, vol. 51, 2015.

[4] J. Bandler, "Have you ever wondered about the engineers mysterious "feel" for a problem?" IEEE Canadian Review, vol. 70, 2013.

[5] T. Simpson, J. Poplinski, N. Koch, and J. Allen, "Metamodels for computer-based engineering design: Survey and recommendations," Engineering with Computers, vol. 17, 2001.

[6] J. Peplinski, K. Allen, and F. Mistree, "Integrating product design with manufacturing process design using the robust concept exploration method," ASME Design Engineering Technical Conferences and Design Theory and Methodology Conference, 1996.

[7] A. Samuelsson, A. C. Bittencourt, K. Saarinen, S. Sander-Tavallaey, M. Norrlöf, H. Andersson, and S. Gunnarsson, "Simulation based evaluation of fault detection algorithms with applications to wear diagnosis in manipulators," IFAC Proceedings Volumes, vol. 47, no. 3, pp. 73677374, 2014.

[8] Ventsim.com - ventsim visual. Accessed: 2016-05-30. [Online]. Available: http://www.ventsim.com/products/ventsim-visual/

[9] Mathworks on matlab. Accessed: 2016-05-31. [Online]. Available: http://se.mathworks.com/products/matlab/

[10] D. Gorissen, K. Crombecq, I. Couckuyt, T. Dhaene, and P. Demeester, "A surrogate modeling and adaptive sampling toolbox for computer based design," Journal of Machine Learning Research, vol. 11, 2010.

[11] F. Pianosi, F. Sarrazin, and T. Wagener, "A matlab toolbox for global sensitivity analysis," Environmental Modelling and Software, vol. 70, 2015.

[12] S. Pope, Turbulent Flows. Cambridge University Press, 2000.

[13] H. Cross, "Analysis of flow in networks of conduits or conductors," University of Illinois at Urbana Champaign, College of Engineering. Engineering Experiment Station., Tech. Rep., 1936.

[14] Ventsim.com - internationally recognised industry standard software. Accessed: 2016-05-30. [Online]. Available: http://www.ventsim.com/internationally-recognised-industrystandard-software/

[15] D. Brake and T. Nixon, "Current trends and future opportunities in underground hardrock ventilation practices," Underground operators conference, 2005.

[16] M. Lundh, J. Nyqvist, and M. Molander, "Deep breaths: Optimizing airflow for underground mines," ABB Review, vol. 2, 2013.

[17] C. Rasmussen and C. Williams, Gaussian Processes for Machine Learning. MIT Press, 2006.

[18] G. Box, J. Hunter, and W. Hunter, Statistics for Experimenters: Design, Innovation, and Discovery, 2nd ed. Wiley, 2005.

[19] M. Thombre, H. Preisig, and M. Addis, "Developing surrogate models via computer based experiments," 12th International Symposium on Process Systems Engineering and 25th European Symposium on Computer Aided Process Engineering, 2015.

[20] D. Gorissen, I. Couckuyt, E. Laermans, and T. Dhaene, "Multiobjective global surrogate modeling, dealing with the 5-percent problem," Engineering with Computers, vol. 26, 2010.

[21] A. Saltelli, M. Ratto, T. Andres, F. Campolongo, J. Cariboni, D. Gatelli, M. Saisana, and S. Tarantola, Global Sensitivity Analysis: The Primer. Wiley, 2008.

[22] Y. Gan, Q. Duan, W. Gong, C. Tong, Y. Sun, W. Chu, A. Ye, C. Miao, and Z. Di, "A comprehensive evaluation of various sensitivity analysis methods: A case study with a hydrological model," Environmental Modelling and Software, vol. 51, 2014.

[23] R. Confalonieri, G. Bellocchi, S. Bregaglio, M. Donatelli, and M. Acutis, "Comparison of sensitivity analysis techniques: A cast study with the rice model warm," Ecological Modelling, vol. 221, 2010. 